\title{
THE NEAR-INFRARED CAPABILITIES OF LEST
}

\author{
ODDBJØRN ENGVOLD \\ Institute of Theoretical Astrophysics, University of Oslo, N-0315 Oslo, Norway
}

\begin{abstract}
The Large Earth-based Solar Telescope (LEST) will be a powerful, next-generation telescope with unprecedented angular resolution, capable of highly accurate polarimetry of the Sun, covering the optical spectral range from about $300 \mathrm{~nm}$ into the near infrared to about $2.5 \mu \mathrm{m}$.

The telescope is a 2.4-m aperture, "polarization-free" concept based on a modified Gregorian optical system. A fast polarization modulator will be located close to the secondary focus of the system. An actively controlled NTT-type main mirror, a high precision pointing and tracking system, a helium-filled light path and a thin entrance window, together with an integrated adaptive optics system, will give the telescope near diffraction-limited performance in the visible. LEST will be sited on La Palma, in the Canary Islands, near the caldera rim on the Roque de los Muchachos Observatory, which often offers excellent seeing. A frequently occurring seeing parameter of $r_{o}=$ $15-20 \mathrm{~cm}$ in the visible will correspond to $r_{0} \geq 1 \mathrm{~m}$ in the near IR.

The construction of LEST will begin in 1993, and the telescope is to be ready for "first light" in 1997. The telescope facility will accommodate a large number of focal plane instruments on a spacious instrument table. LEST will be made available for near-IR instrumentation from the start of its regular operation.
\end{abstract}

Key words: infrared: stars - instrumentation: polarimetric - telescopes - Sun: general

\section{Introduction}

\subsection{INTRODUCTORY REMARKS}

The Large Earth-based Solar Telescope (LEST) is not a telescope that has been designed for the infrared; yet, it will offer interesting opportunities for observations in the near-infrared out to wavelengths $\lambda \sim 2.5 \mu \mathrm{m}$. LEST will be a unique facility for near-IR observations of the Sun because of its ( $i)$ large (2.4-m) light-collecting aperture (high photon flux), (ii) diffraction limited performance $\left(\theta \leq 0.25^{\prime \prime}\right.$ in the near IR), and (iii) good polarimetric qualities.

\subsection{Science With The LEST}

The unceasing scientific demand for yet sharper and more precise imaging of smallscale processes in the solar atmosphere is behind the joint international efforts to develop and build a next-generation, high-resolution solar observing facility. Observational and theoretical discoveries suggest that much of the dramatic variability of the Sun is tied to small-scale processes involving the magnetic fields in the solar atmosphere (MacQueen 1987). For instance, how does the presence of a magnetic field modify convection, and how does fluid motion alter the structure of the magnetic field? How is the fine-scale magnetic structure of the Sun's atmosphere related to the heating of the outer layers and to various forms of activity? Being the only star close enough for study of many of its phenomena and underlying processes, the Sun can be perceived as the key to understanding the physics of other stars.

We have been reminded by several speakers at this conference about the many unique and exciting possibilities in the infrared and near-infrared for diagnostics of the solar photosphere. Seeing is the basic limitation to spatial resolution in the visible, while at a good site resolution in the near IR may be telescope limited 
even for apertures exceeding $1 \mathrm{~m}$. While in the visible one obtains magnetic field strengths by indirect methods (Stenflo 1989), in the near IR some lines are fully split (Solanki 1993) and thus provide direct measurements of magnetic field strengths. The richness of photospheric molecular and atomic lines in the IR, especially in spectra of sunspot umbrae, is demonstrated nicely by the new spectrum atlas of Livingston and Wallace (1991). In the near IR, one takes advantage of the minimum absorption around $1.6 \mu \mathrm{m}$ to probe the deeper layer of the photosphere. The deep layers of the granulation and magnetic flux elements may be accessed here with high spatial resolution (Stenflo et al. 1987; Koutchmy 1993).

\section{The LEST Project}

\subsection{An International Cooperation in Solar Physics}

The ultimate realization of LEST, the world's largest solar telescope on a superb site in the Canary Islands, is expected to provide the global solar physics community with a next-generation scientific facility which will be uniquely able to tackle from the ground the difficult and pressing observational tasks in solar research for the 1990 's and well into the 2000's.

The well-advanced development of the LEST project is being recognized in several national planning reports on future astronomical facilities. In the listing of future astronomical facilities by the Deutsche Forschungsgemeinschaft, the LEST project ranked high among projects recommended for national funding. In the report from the Joint Working Group on Ground-based Astronomy, which involved representatives from national research councils of Canada, France, Germany, Italy, Japan, United Kingdom, and the United States, the assessment was that "the LEST project is one of major importance for the solar physics community and one which can proceed only by international collaboration. The report Astronomy in the U.S.A. for the 1990's (Bahcall Report) and the European Physical Society (EPS) Report Solar Physics in Europe recommended a strengthening of solar ground-based research facilities. The priority for the project is very high within most of the countries of the LEST consortium."

\subsection{Organization}

The LEST Foundation is a non-profit organization created in 1983 to serve this major international activity and collaboration in solar physics and astronomy (Wyller 1991). It is domiciled, under Swedish law, in The Royal Swedish Academy of Sciences in Stockholm, and now comprises member organizations from nine nations; Germany, Israel, Italy, Norway, Spain, Sweden, Switzerland, and USA.

\subsection{The Site of LEST in the Canary Islands}

The LEST site is located on La Palma, in the Canary Islands, at altitude 2,350 m above sea level, in the neighborhood of the Swedish 50-cm optical solar telescope and a number of nighttime telescopes (Wyller 1991). The high optical quality of the La Palma site is well documented from extensive experience with the Swedish 
vacuum tower telescope, the site of which is practically identical to the LEST site. The image quality achieved with this telescope is widely recognized to be superior to that of any other solar telescope in the World, frequently providing diffraction limited performance with its 50-cm aperture (Wyller and Scharmer 1985; Scharmer 1989). This indicates the uniqueness of the site: The conditions for high-resolution observations are favorable, and the La Palma site satisfies the scientific requirements for a next-generation type 2.4-m telescope like LEST.

\section{A Design for High Resolution Observation of the Sun with High Polarimetric Accuracy}

\subsection{Design Specifications}

LEST is conceived as a comprehensive facility for observations of the Sun in the visible and near-infrared, with angular resolution $\theta \leq 0.1^{\prime \prime}$ in the visible (which will require an aperture $\geq 2 \mathrm{~m}$ ) and low instrumental polarization in order to measure polarization to an accuracy $\Delta p / p \approx 10^{-4}$. The large aperture will be needed also to ensure a high photon flux and thereby short integration times for measurements of small-scale solar structures. For more details, see Engvold and Andersen (1990a). Being a ground-based facility to serve a large international community, LEST will offer flexibility and ample space for a variety of both permanent and experimental post-focus instruments.

\subsection{Development of the LEST Design}

The design of LEST and its new-technology enclosure, is the result of extensive studies involving experts from participating institutes. The results from many such studies are described in the LEST Technical Report series, which presently count 54 reports. On the basis of a technical concept developed by Andersen et al. (1984), and the management plan by Engvold and Hillerud (1988), the LEST Foundation decided to contract a major part of the design work to the engineering team located at Ris $\varnothing$, Denmark, that previously had designed and built the Nordic Optical Telescope in La Palma, in addition to doing consulting work for ESO's VLT. The LEST design, as described in some detail below, was adopted by the LEST Foundation in 1990 (Engvold and Andersen 1990a,b). Table I summarizes some of the basic design parameters.

\subsection{The Telescope}

The LEST telescope is a modified Gregorian type with an additional concave mirror behind the primary. More than $99 \%$ of the energy is absorbed by a heat-rejector at the primary focus of the parabolic $\mathrm{f} / 2.3$ main mirror. The third mirror forms an $f / 75$ beam, and re-images the Gregorian focus at the large instrument table at the base of the telescope. The same mirror also forms an image of the pupil at the position of the flat M5, which will be a fast, agile guider mirror of this system. For the adaptive optics system of the LEST, the adaptive mirror will be inserted immediately above M5. Figure 1 shows the optical layout of the LEST. 
TABLE I

Technical Data on LEST

\begin{tabular}{|l|c|}
\hline Design Parameters & \\
\hline \hline Pupil diameter & $240 \mathrm{~cm}$ \\
Focal length (effective) & $176.5 \mathrm{~m}$ \\
f/D & 73.5 \\
Diffraction limited & $0.06^{\prime \prime}-0.25^{\prime \prime}$ \\
resolution & (visible - near IR) \\
Image scale & $1.17 \mathrm{arcsec} \mathrm{mm}^{-1}$ \\
Entrance window & $\varnothing 250 \mathrm{~cm} \times 30 \mathrm{~mm}$ \\
(fused silica) & \\
Wavelength range & $0.3-2.5 \mu \mathrm{m}$ \\
Field of view & $\pm 1^{\prime}$ \\
(non-rotating) & $\geq 15$ \\
Number of exit ports & $\sim 30 \mathrm{~m}$ \\
Tower height & \\
\hline
\end{tabular}

\subsection{INSTRUMENT TABLE}

A large, 19.5-m-diameter, rotating platform at the bottom of the tower provides adequate space for the LEST focal plane instruments (see Fig. 2). A 12-m deep, steel cylinder in the center of the instrument table provides space for large verticallymounted instrumentation. Full compensation for field rotation is achieved through proper relative motion of the table and the mirror M6 (see Fig. 1).

\subsection{Tower and Dome Structure}

The compact telescope and integrated mounting of LEST is placed on top of a single conically-shaped, concrete tower. The tower is about $30 \mathrm{~m}$ tall, to minimize the influence of the ground turbulence. The compact, light-weight, spherical dome and tube protect the telescope from radiative heating and weather, and the aerodynamical shape serves to reduce wind buffeting. A cross section of tower and dome is seen in Figure 2.

\subsection{Pola Rimetry}

Because measurements of magnetic fields is a main scientific objective of LEST, the photon flux requirements of Stokes polarimetry have been a fundamental design consideration ( $c f$. Lites $1987 a$ ). The polarization modulator of the polarimetry system will be inserted into the light beam prior to the first inclined reflection. Its location is immediately before the secondary image plane of the modified Gregorian telescope. A high frequency $(50-100 \mathrm{kHz})$ piezo-elastic modulator (PEM) will be used in conjunction with a demodulation scheme based on synchronous shifting of charges in a modified CCD in the instrument focus. The proposed system is being 


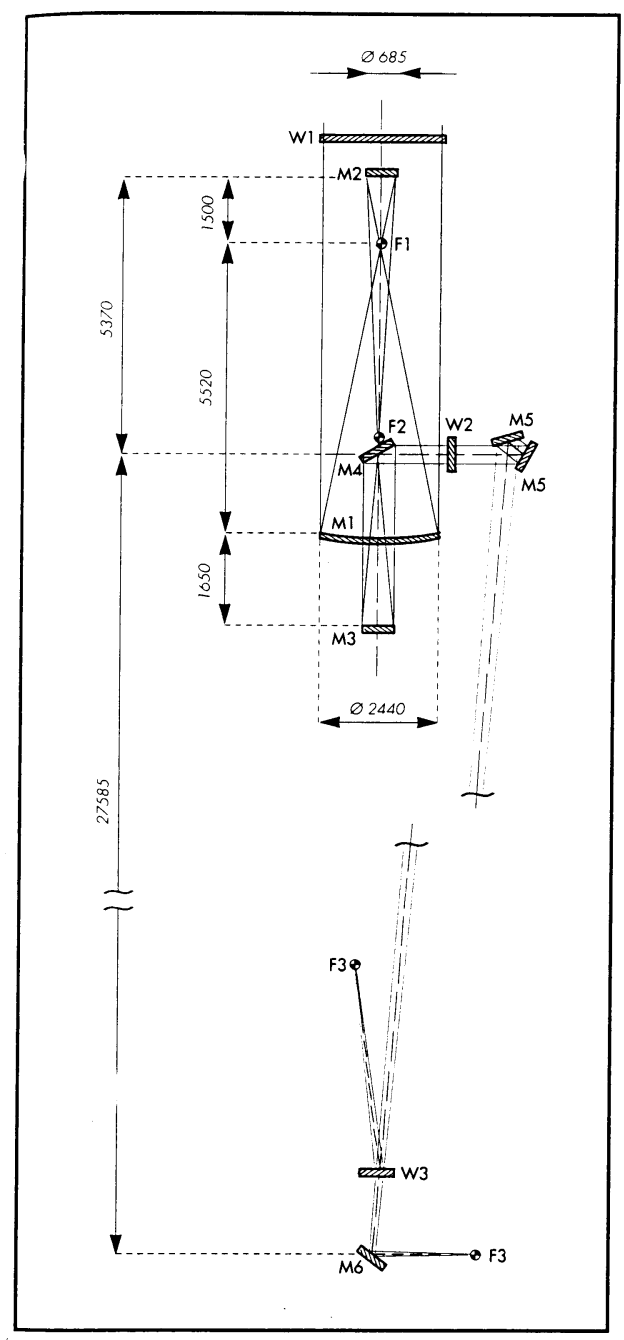

Fig. 1. Layout of the optical systems of LEST.

developed by Keller et al. (1989). An alternative modulation scheme is discussed by Lites $(1987 b)$.

The "straw-man" modulator package performs without problems over the whole near-IR wavelength range. Demodulation at the exit focus cannot, however, be done with the charge-shifting technique in the CCD chip, as foreseen for the observations in the visible, since IR-sensitive CCD's with this type of architecture are not yet available. For the near IR an optical demodulation scheme based on available technology is proposed, as described by Stenflo and Povel (1985) and Stenflo (1991). 


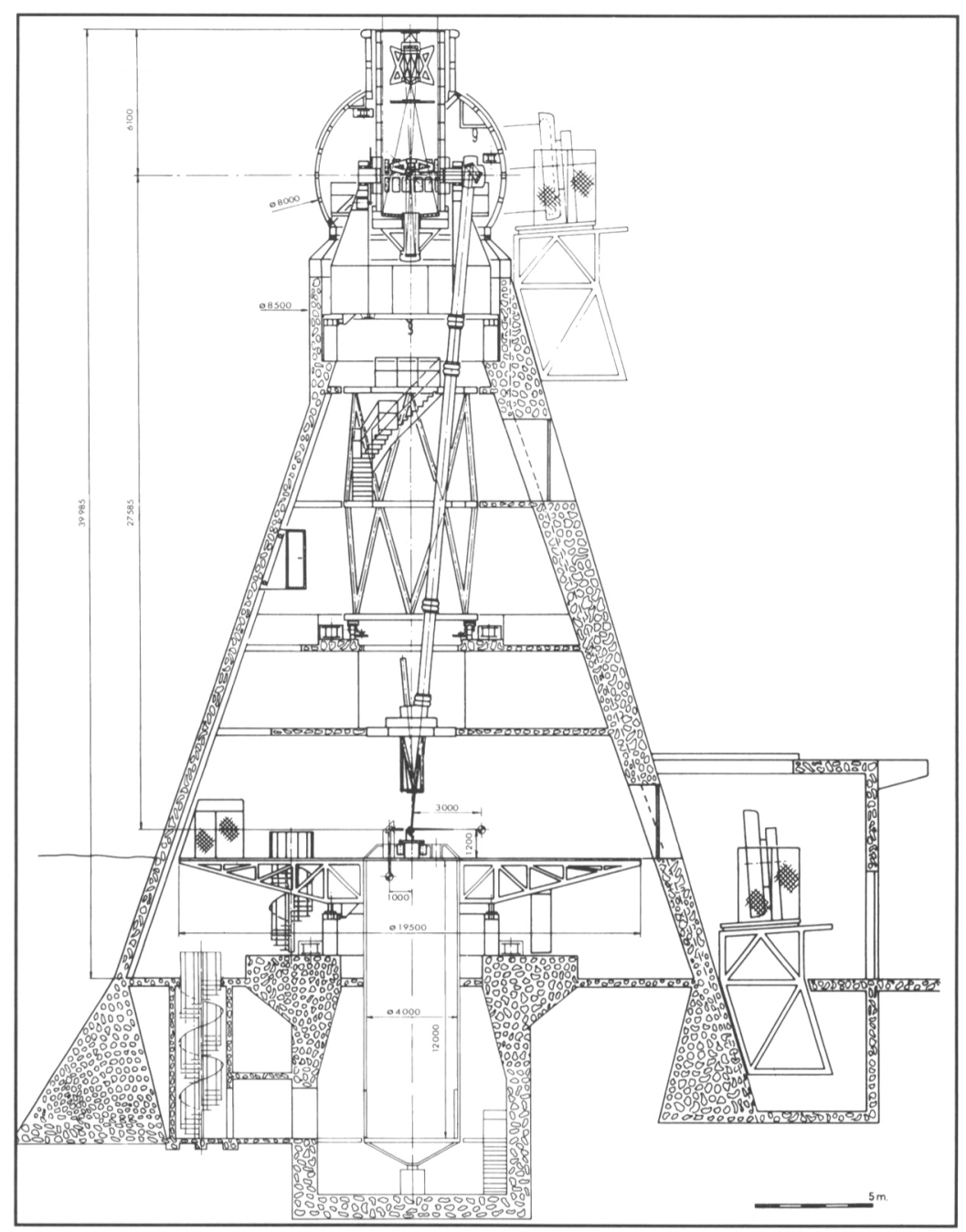

Fig. 2. Cross section of the telescope and tower structure of LEST.

\subsection{Entrance Window}

Most modern solar telescopes have an evacuated light path to eliminate internal seeing (Dunn 1985). However, the thermal and mechanical stresses in the entrance windows of vacuum telescopes are problematical and reasons for concern, particularly for polarimetric observations (Dunn 1984; Owner-Petersen 1991). Using a vacuum window will, in practice, limit the telescope aperture to less than $1 \mathrm{~m}$, since the necessary thickness would otherwise be unacceptable with regard to optical quality. The idea of filling LEST with helium was explored through several practical studies (Engvold et al. 1983), and more recently by measurement in a 

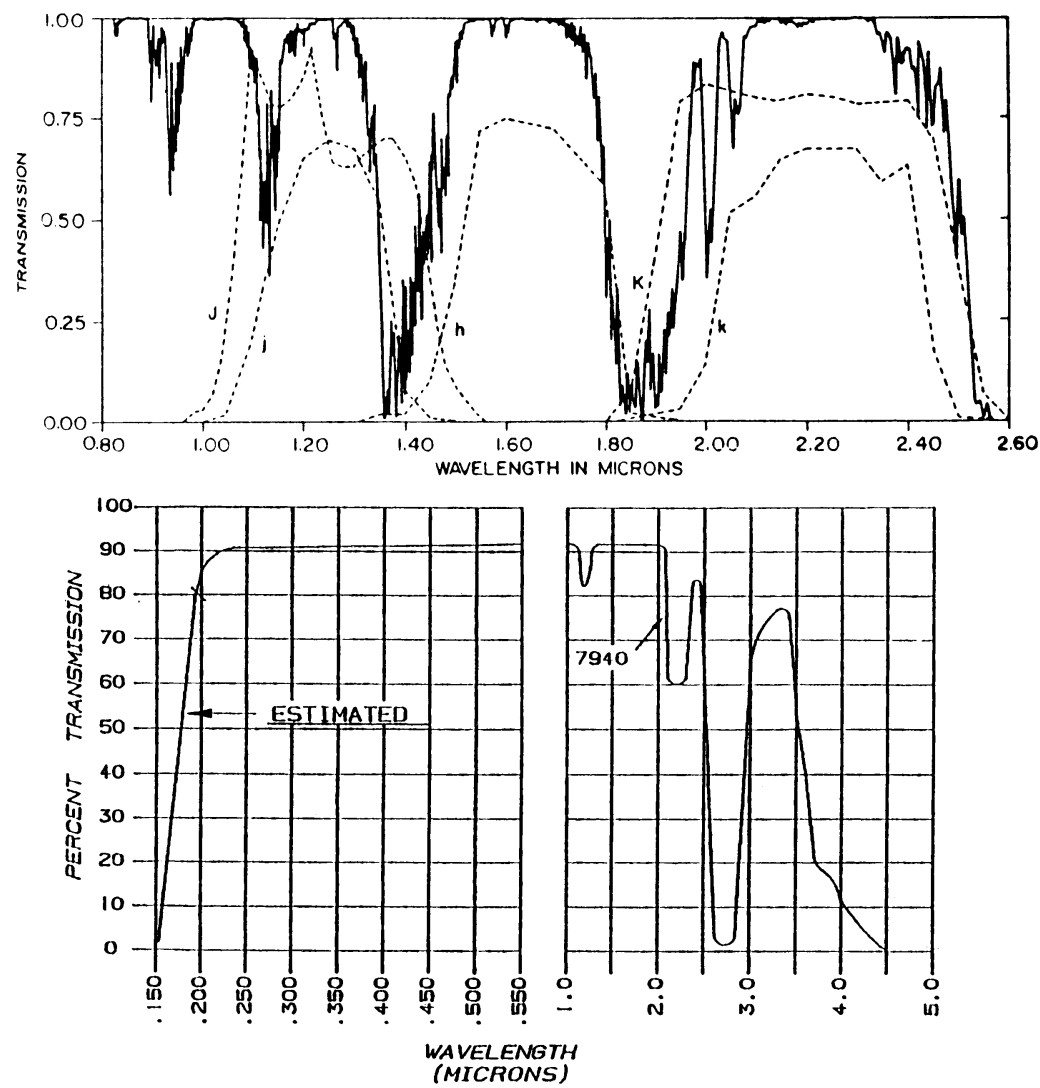

Fig. 3. Upper diagram: Optical transmission of the atmosphere in the near IR. Lower diagram: Optical transmission of a 1-cm thick window of fused silica, including surface losses.

full-scale model of the LEST telescope tank (Engvold and Andersen 1990a). These measurements have demonstrated that a circulating helium atmosphere in the tank, which allows for the use of a thin, high-quality optical window, eliminates the problem of telescope seeing.

The shortest wavelengths accessible to LEST will be limited by the atmosphere $(\lambda \sim 300 \mathrm{~nm})$ and the longest wavelengths by absorption in the entrance window $(\leq 2.5 \mu \mathrm{m})$ (Fig. 3). The scientific gain from making LEST available for observations at wavelengths beyond $\sim 2.5 \mu \mathrm{m}$ is unquestionable. However, removing the entrance window would lead to a number of new problems. Exposure to weather and dust in the atmosphere would inevitably harm the many delicate components and high-quality optics inside the tube and, thus, severely impair the use of LEST in the visible. Moreover, mounting and dismounting of the fragile window would be extremely risky and difficult. For these reasons the LEST will not be used without its window. 


\subsection{High-Resolution Imaging}

The successful implementation of an active figure-controlled main mirror of ESO's NTT (Wilson 1990) has suggested use of a similar meniscus-type main mirror for LEST. The LEST main mirror will be supported axially by 81 individually regulated points and 3 actuators to control tilt, and in the transversal direction by 24 individually regulated points and again 3 actuators.

The tight positional and alignment tolerances of the primary and secondary mirrors call for a continuous monitoring and correcting system for LEST. For this purpose one will use a low spatial and temporal resolution Hartmann-Shack type wavefront sensor with correlation trackers. The wavefront sensor will use light reflected off the small exit window, W3, near focus F3 (see Fig. 1). It is foreseen that this system will be used to sense and update the mirror alignment, and to check the main mirror figure once every several minutes, without interfering with observations.

Adaptive optics (AO) systems are currently becoming available for astronomy. There is little doubt that an AO system will be available for LEST, the question is rather when. Recent tests made with the vacuum tower telescope at Sacramento Peak, using the AO system of the Lockheed group, have shown promising results (Acton 1990). An AO system will be an integral part of the LEST telescope ( $c f$. Dunn 1987). The deformable mirror and the fast tilt-mirror will be located at the image of the pupil on the elevation axis outside the telescope tube.

LEST will be unsurpassed for high-resolution observations also in the near IR. A frequently-occurring seeing parameter $r_{o}=15-20 \mathrm{~cm}$ in the visible corresponds to $r_{o} \geq 1 \mathrm{~m}$ in the near IR. With the good seeing quality of the La Palma site, LEST is expected to give near diffraction-limited imaging in the near IR, even without an adaptive optics system. Also, an AO system is going to work better in the near IR than in the visible, because of the more relaxed precision required at longer wavelengths.

\section{Observing with LEST in the Near-Infrared}

The high $S / N$ made possible by the large aperture of LEST will allow the measurement of weak magnetic fields using ratios between different spectral lines in the near IR. The Stokes V signal is smaller in the near IR, since the lines are generally weaker and broader, and therefore a large aperture is needed. A high $S / N$ ratio is also required to study granulation in the near IR, since the granulation contrast there is lower than in the visible.

Stokes $Q, U$, and $V$ tend to be comparably strong in the IR (when there is complete Zeeman splitting of the lines). Therefore, any cross-talk that may occur between them (due to instrumental polarization) would be disastrous. The polarization-free property of the LEST is thus even more important in the IR than it is in the visible.

The use of the same modulator package at the LEST secondary focus for both the visible and near IR presents a fundamental advantage by allowing for simultaneous, or nearly simultaneous, observations in the visible and near IR. This is 
important, since the diagnostic information in these two wavelength regions are complementary and refer to different heights in the solar atmosphere. Such observations could allow the 3 -D mapping of magnetic, thermal and dynamic structure of small-scale flux tubes.

In summary, LEST will offer the following features and possibilities for observations at near-IR wavelengths:

- Spatial resolution of 0.15-0.25".

- $\quad$ High $S / N$, made possible by large collecting aperture.

- The same polarization modulation package can be used in the visible and near IR, allowing nearly simultaneous observations in these two $\lambda$-regions.

- Fully split Zeeman lines for measurements of network elements (1200-1500 G).

- Low instrumental polarization - eliminating cross talk between Stokes $Q, U$, and $V$, which are comparably strong in the IR.

- Thermal control of outer and inner structures.

- $\quad$ Adequate space for a large number of focal-plane instruments.

\section{Concluding Remarks}

The Design Study has been reviewed and approved by the LEST Foundation, and the technical group responsible for this study is primed to embark upon the final Construction Phase of the LEST Telescope. With the requisite funding by various national agencies, the Construction Phase will begin in the fall of 1993 with a completion date in 1997.

The LEST Foundation will build the "core" telescope as described in Section 3. The telescope will be furnished with a polarimetry system, and later an AO system, in addition to such facilities as a high-resolution spectrograph and a universal narrow-band filter. The large instrument table and central tube provide space and focal-plane ports for a large number $(\geq 15)$ of permanent and experimental set-ups. Standardized specification and interfacing will enable LEST members and other groups to bring and temporarily use a number of specialized instruments.

Since there is a large potential at LEST for near-IR research right from the start, due to less stringent requirements in optical precision and seeing conditions, the development of a near-IR spectrograph for LEST will be given high priority.

\section{Acknowledgements}

Helpful suggestions and input for this talk were given by Jan Olof Stenflo and Mette Owner-Petersen.

\section{References}

Acton, D.S.: 1990, Real-Time Solar Imaging with a 19-segment Active Mirror System, Ph.D. Thesis, Texas Tech University.

Andersen, T.E., Dunn, R.B., and Engvold, O.: 1985, LEST Technical Report No. 7.

Dunn, R.B.: 1984, LEST Technical Report No. 3 .

Dunn, R.B.: 1985, Solar Phys. 100, 1.

Dunn, R.B.: 1987, LEST Technical Report No. 28, p. 243.

Engvold, O., Dunn, R.B., Livingston, W.C., and Smartt, R.: 1983, Appl. Optics 22, 10. 
Engvold, O., and Hillerud, K.-I.: 1988, LEST Technical Report No. 29.

Engvold, O. and Andersen, T.: 1990a, LEST Design, Report of the LEST Foundation issued August 1990.

Engvold, O. and Andersen, T.: 1990b, LEST Technical Report No. 42.

Keller, C.U., Aebersold, F., Egger, U., Povel, H.P., Steiner, O., and Stenflo, J.O.: 1993, LEST Technical Report No. 53.

Koutchmy, S.: 1993, these proceedings.

Lites, B.W.: 1987a, LEST Technical Report No. 22.

Lites, B.W.: 1987b, LEST Technical Report No. 23.

Livingston, W. and Wallace, L.: 1991, An Atlas of the Solar Spectrum in the Infrared from 1850 to $9000 \mathrm{~cm}^{-1}$ (1.1 to $\left.5.4 \mu \mathrm{m}\right)$, NSO Technical Report No. 91-001.

MacQueen, R.M.: 1987, LEST Technical Report No. 24.

Owner-Petersen, M.: 1991, LEST Technical Report No. 49.

Scharmer, G.B.: 1989, in R.J. Rutten and G. Severino (eds.), Solar and Stellar Granulation, NATO ASI Series, Kluwer Academic Publishers, p. 161.

Solanki, S.K.: 1993, these proceedings.

Stenflo, J.O.: 1989, Astron. Astrophys. Rev. 1, 3.

Stenflo, J.O.: 1991, LEST Technical Report No. 44.

Stenflo, J.O. and Povel, H.P.: 1985, LEST Technical Report No. 12.

Stenflo, J.O., Solanki, S.K., and Harvey, J.W.: 1987, Astron. Astrophys. 173, 167.

Wilson, R.N.: 1990, The Messenger, ESO, No. 59, p. 7.

Wyller, A.A., Scharmer, G.B.: 1985, Vistas in Astronomy 28, 467.

Wyller, A.A.: 1991, LEST - An International Solar Telescope, Pub. of the LEST Foundation. 